\title{
Formulation, characterization, and expression of a recombinant MOMP Chlamydia trachomatis DNA vaccine encapsulated in chitosan nanoparticles
}

This article was published in the following Dove Press journal:

International Journal of Nanomedicine

7 May 2013

Number of times this article has been viewed

\author{
Chino D Cambridge \\ Shree R Singh \\ Alain B Waffo \\ Stacie J Fairley \\ Vida A Dennis
}

Center for NanoBiotechnology

Research (CNBR), Alabama State

University, Montgomery, AL, USA
Correspondence: Vida A Dennis

Department of Biological Sciences,

$\mathrm{PhD}$ program in Microbiology,

Alabama State University,

1627 Hall Street, Room 307,

Montgomery, AL 36104, USA

Tel + I 3342298447

Email vdennis@alasu.edu
Abstract: Chlamydia trachomatis is a bacterial sexually transmitted infection affecting millions of people worldwide. Previous vaccination attempts have employed the recombinant major outer membrane protein (MOMP) of C. trachomatis nonetheless, with limited success, perhaps, due to stability, degradation, and delivery issues. In this study we cloned C. trachomatis recombinant MOMP DNA (DMOMP) and encapsulated it in chitosan nanoparticles (DMCNP) using the complex coacervation technique. Physiochemical characterizations of DMCNP included transmission and scanning electron microcopy, Fourier transform infrared and ultravioletvisible spectroscopy, and zeta potential. Encapsulated DMOMP was 167-250 nm, with a uniform spherical shape and homogenous morphology, and an encapsulation efficiency $>90 \%$. A slow release pattern of encapsulated DMOMP, especially in acidic solution, was observed over 7 days. The zeta potential of DMCNP was $\sim 8.80 \mathrm{mV}$, which indicated that it was highly stable. Toxicity studies of DMCNP $(25-400 \mu \mathrm{g} / \mathrm{mL})$ to Cos-7 cells using the MTT assay revealed minimal toxicity over 24-72 hours with $>90 \%$ viable cells. Ultra-violet visible (UV-vis) spectra indicated encapsulated DMOMP protection by chitosan, whereas agarose gel electrophoresis verified its protection from enzymatic degradation. Expression of MOMP protein in DMCNPtransfected Cos-7 cells was demonstrated via Western blotting and immunofluorescence microscopy. Significantly, intramuscular injection of BALB/c mice with DMCNP confirmed the delivery of encapsulated DMOMP, and expression of the MOMP gene transcript in thigh muscles and spleens. Our data show that encapsulation of DMOMP in biodegradable chitosan nanoparticles imparts stability and protection from enzymatic digestion, and enhances delivery and expression of DMOMP in vitro and in mice. Further investigations of the nanoencapsulated DMCNP vaccine formulation against $C$. trachomatis in mice are warranted.

Keywords: Chlamydia trachomatis, bacteria, chitosan, DNA, MOMP, vaccine

\section{Introduction}

Chlamydia trachomatis is the leading cause of bacterial sexually transmitted infections in both developed and developing countries, ${ }^{1}$ with an estimated 90 million reported new cases annually. ${ }^{2}$ Prolonged infection with $C$. trachomatis often leads to other complications such as pelvic inflammatory disease (PID), ectopic pregnancy, infertility, and chronic abdominal pain. ${ }^{3}$ In addition, enhancement of the human immunodeficiency virus (HIV) transmission can occur due to the presence of a C. trachomatis infection. ${ }^{4}$ C. trachomatis infections and associated complications amass expenses in excess of US\$10 billion annually, ${ }^{5}$ and are a significant socioeconomic burden.

Antibiotic regimens are effective for treatment of $C$. trachomatis infections, albeit with limitations, because once infection has ensued and becomes chronic, treatment of 
the bacteria may prove futile leading to reinfection. Hence, development of a vaccine formulation is a more promising and effective approach for controlling C. trachomatis. Attempts at vaccine formulation have been underway, predating the 1970s where whole organisms were tested against different serovars. ${ }^{6}$ However, protection gained from the whole organism vaccine was short-lived and strain specific. ${ }^{6}$ Thus, as yet there is still no approved vaccine against $C$. trachomatis.

Progression in C. trachomatis analysis led to the characterization of several surface exposed proteins in particular its major outer membrane protein (MOMP). Determination of MOMP as a structurally ${ }^{7}$ and immunodominant ${ }^{8}$ protein of $C$. trachomatis put it at the forefront of being the best understood and most desirable vaccine candidate. MOMP is a $40 \mathrm{kDa}$ cysteine rich protein with numerous immunogenic $\mathrm{B}$ - and T-cell epitopes and protective antigens, ${ }^{7,9-13}$ thus making it ideally suited and attractive as a vaccine candidate. The native form of MOMP reportedly elicited a protective immune response to a $C$. trachomatis genital challenged infection in mice, which was similar to that elicited by live elementary bodies. ${ }^{14}$ However, a native MOMP vaccine is not practical because of the cost associated with its mass production. Therefore, recombinant MOMP (rMOMP) has been widely employed in vaccine studies but protection attained in efficacy studies is not as robust as that of native MOMP. ${ }^{15}$ Another barrier faced in the use of MOMP in vaccine formulation is its rapid degradation via proteases, which often leads to the poor cellular uptake of MOMP and thus a reduction in its immunogenic capacity. ${ }^{16,17}$ As a result of this instability of MOMP, vaccine formulations against C. trachomatis also now target DNA-based systems.

DNA vaccines, like their protein counterpart, possess the ability to induce both cellular and humoral immune responses as demonstrated for a variety of pathogens. ${ }^{18,19}$ Long-term persistence of the presented immunogen is also achieved via DNA vaccination. Other benefits of a DNA-based vaccine include its ability to polarize T-cell help, especially to a Th1 immunological response, as well as ease in the burden of production, compared with a protein-based vaccine. DNA vaccines are also beneficial through the extension in shelf-life gained (storage and shipping capacity), as well as through vaccine stability, providing yet again a less expensive means of manufacturing.

A major hurdle in development of a vaccine against C. trachomatis is an effective delivery system for either a proteinor DNA-based immunogen. Although several delivery systems have been employed in C. trachomatis vaccine development projects, ${ }^{9,13,20}$ they have not been successful in rendering complete protection against this pathogen. An immunogen, coupled with an effective vaccine delivery system, appear to be paramount in potentially achieving complete protective immunity against $C$. trachomatis. To this end, nanoparticles have emerged as novel delivery vehicles for vaccination against many pathogens, ${ }^{21,22}$ including C. trachomatis..$^{20,23}$ Notably amongst these nanoparticles is chitosan, a cationic polymer with favorable biological properties. ${ }^{24}$

Chitosan is generated from the deacetylation of chitin, the structural component in the exoskeleton of crustaceans. The deacetylated chitosan backbone of glucosamine units has a high density of amine groups, permitting strong electrostatic interactions with proteins and genes, which carry overall negative charge at neutral $\mathrm{pH}$ conditions..$^{25,26}$ Chitosan is relatively non-toxic and biodegradable ${ }^{27}$ with a high charge density. ${ }^{28}$ This along with its low level of toxicity, high encapsulate capacity, ${ }^{21}$ mucoadhesiveness, and drug penetration enhancement capacity across mucosal barriers, ${ }^{29}$ make it very attractive in the realm of vaccine delivery development. Chitosan can be degraded into N-acetyl-glucosamine by general body lysozymes, which subsequently is excreted as carbon dioxide via the glycoprotein synthetic pathway. ${ }^{30}$

In the present study, we formulated a DNA vaccine encoding for MOMP of C. trachomatis (DMOMP) and encapsulated it in chitosan nanoparticles (DMCNP) using the complex coacervation technique. The DMCNP was subjected to physiochemical characterizations including Fourier transform infrared and ultra-violet (UV) spectrophotometry to verify encapsulation; and zeta potential and electrophoresis mobility analyses for stability determinations followed by transmission and scanning electron microscopy for morphology and size. Next, we investigated the toxicity of DMCNP on Cos-7 cells, and the in vitro release of encapsulated DMOMP from DMCNP. Expression of MOMP protein in Cos-7 cells transfected with DMCNP was verified by Western blotting and immunofluorescence microscopy. Finally, in vivo expression of the MOMP gene transcript in muscle tissues and spleens of mice injected with DMCNP was accomplished by reverse transcription polymerase chain reaction (RT-PCR). Here we present and discuss our results in the context of chitosan nanoparticles as an effective delivery system for DMOMP.

\section{Methods and materials Materials}

The phCMV1 vector, containing the intron A from the human CMV IE (immediate-early) gene was purchased 
from Genlantis, (San Diego, CA, USA). Restriction enzymes (BamH I, Not I and Pst I) were purchased from New England Bio-labs (Ipswich, MA, USA); donkey anti-goat IgG $(\mathrm{H}+\mathrm{L})$ was purchased from Invitrogen (Carlsbad, CA, USA). Medium molecular weight chitosan and chitosanase (Streptomyces griseus) were obtained from Sigma-Aldrich (St Louis, MO, USA). Polyclonal antibodies against $C$. trachomatis (MOMP) were purchased from Fitzgerald Industries International (Acton, MA, USA), with FITC rabbit anti-goat antibodies IgG $(\mathrm{H}+\mathrm{L})$ being purchased from Southern Biotech (Birmingham, AL, USA). Plasmid DNA preparation kits, as well as DNA gel extraction kits were purchased from Qiagen (Valencia, CA, USA). Amaxa Cell Line Nucleofector Kit was purchased from Lonza (Walkersville, MD, USA).

\section{Plasmid phCMVI-MOMP construction for DNA vaccine}

The MOMP gene of $C$. trachomatis was amplified from the plasmid pET-MOMP $^{9}$ by polymerase chain reaction (PCR) and cloned into the phCMV1 vector. Primers used for PCR amplification were phCMV1 MOMP BamH I Dir (for forward) and phCMV1 MOMP Not I Rev (for reverse) with the respective sequences: 5'GGATCCACCATGGCTTCCTCCTTGCATGCTCTGCCTG3', and 5'CCACCGCGGCCGCTCATtAaGtAaGtCGACGGAACTGAGCA3'. Phusion high-fidelity DNA polymerase protocol was used to amplify the MOMP gene with the optimized forward primer containing a Kozak consensus sequence and unique cloning restriction enzyme site (BamH I). The reverse primer also contained Not I unique and two different stop codons used to ensure the expression of only the full length MOMP protein. The PCR product was analyzed by gel electrophoresis for the correct size and then subjected to unique restriction enzyme digestion using BamH I and Not I. Likewise, the phCMV1 vector was subjected to the same restriction enzyme digestion. Both the MOMP gene and phCMV1 vector were purified by low melting agarose electrophoresis, quantified prior to ligation, and then used to transform competent Escherichia coli MC1061. Transformed bacteria were selected on solid media containing kanamycin, clones were screened using previous cloning restriction enzymes and Pst I, and then confirmed by sequencing. A recombinant clone with the appropriate sequenced MOMP gene (DMOMP) was scaled using Qiagen Endo-free Giga kit (Valencia, CA, USA) and used for the entire study.

\section{Chitosan purification}

Chitosan preparation and purification were achieved following previous methods ${ }^{21}$ with modifications.
Chitosan flakes $(1 \mathrm{~g})$ were dissolved in $15 \mathrm{~mL}$ of $1 \mathrm{M} \mathrm{NaOH}$ and heated for 2 hours at $50^{\circ} \mathrm{C}$ with continuous stirring. The resulting solution was filtered, washed with deionized water and dried overnight at $40^{\circ} \mathrm{C}$. Dried chitosan flakes were dissolved in $0.1 \mathrm{M}$ acetic acid, filtered using a Buchner filtration unit (Fischer Scientific, Houston, TX, USA), adjusted to $\mathrm{pH} 8.0$, ultimately resulting in the formation of purified chitosan precipitates. Chitosan precipitates were collected by centrifugation, vacuum-dried for 20 hours at room temperature, and then dissolved in $1 \%$ acetic acid to obtain a $2 \%$ chitosan solution.

\section{Preparation of DMOMP in chitosan nanoparticles}

DMOMP was prepared in chitosan nanoparticles using the complex coacervation method ${ }^{21}$ with minor modification. The chitosan solution consisted of $0.2 \mathrm{~mL}$ of $2 \%$ chitosan, in combination with $0.8 \mathrm{~mL}$ of $5 \mathrm{mM}$ sodium acetate ( $\mathrm{pH} 5.5)$. The DNA solution consisted of $0.4 \mathrm{~mL}$ of DMOMP and $3.6 \mathrm{~mL}$ of $45 \mathrm{mM}$ sodium sulfate. Both solutions were heated to $55^{\circ} \mathrm{C}$ for 15 minutes in a water bath. A ratio of $1: 3$ chitosan to DNA solution was obtained by adding the chitosan solution drop-wise to the DNA with gentle agitation for 15-25 seconds thus resulting in a spontaneous nanoparticle formation (DMCNP). Phosphate buffered saline (PBS) was encapsulated in chitosan nanoparticles (CNP) to serve as a negative control. Both DMCNP and CNP were stored at $4^{\circ} \mathrm{C}$ until used.

\section{Encapsulation efficiency and in vitro release analyses}

Encapsulation efficiency of DMCNP was determined by sucrose gradient ultracentrifugation as described ${ }^{21}$ using the formula: Encapsulation Efficiency $=\mathrm{A}-\mathrm{B} / \mathrm{A} \times 100$ where (A) is the total sum of DNA loaded and, (B) is free DNA.

The analysis of DMOMP released from DMCNP was evaluated using similar intestinal fluid (SIF, $\mathrm{pH}$ 7.0) and similar gastrointestinal fluid (SGF, pH 2.0) essentially as published. ${ }^{21}$ In addition, samples were incubated in PBS ( $\mathrm{pH}$ 7.4) and incubated at $37^{\circ} \mathrm{C}$. Samples were collected at varying time-intervals (day $0.08,1,2,4,5$, and 7) and the released DNA samples were measured using NanoDrop ND1000 (Thermo Scientific, Rockford, IL, USA) at $260 \mathrm{~nm}$.

The released products from each time-point were precipitated with $5 \mathrm{M} \mathrm{NaCl}$ and amplified by PCR as described above and amplicons subjected to agarose gel electrophoresis for verification of the specific DMOMP product. The resulting products were confirmed as DMOMP 
in reference to the positive DMOMP clone with an expected 1154 base pair (bp) size.

\section{Protection assessment of DMCNP}

The integrity of protection of encapsulated DMOMP in chitosan nanoparticles was validated using previously described methods ${ }^{21,31}$ with some modification. Both DMCNP and DMOMP (1 $\mathrm{mg} / \mathrm{mL})$ were subjected to enzymatic digestion with Nde I and Sal I in combination with, or without chitosanase for 2 hours at $37^{\circ} \mathrm{C}$ according to the manufacturer's protocol. For DNAase 1 analysis, DMOMP or DMCNP suspensions were incubated in DNAase 1 for 15 minutes at $37^{\circ} \mathrm{C}$ and the reaction was stopped by the addition of iodoacetic acid (a DNAase 1 inhibitor). All samples were analyzed on a $0.8 \%$ agarose gel for the presence or absence of DNA or nanoparticles. Corresponding bands were visualized with a ChemiImager gel documentation apparatus (Alpha Innotech Corp, San Leandro, CA, USA).

\section{Stability studies}

Stability assessment of DMCNP was established following previously described methods. ${ }^{21}$ DMCNP was added to individual micro-centrifuge tubes and adjusted to various $\mathrm{pH}$ values $(2,4,6,8,9,10,10.5$, and 12) followed by incubation at $37^{\circ} \mathrm{C}$ on a shaker for 30 minutes. All samples were analyzed by agarose gel electrophoresis and visualized using the Chemilmager ${ }^{\mathrm{TM}}$ gel documentation system (Genetic Technologies, Inc, Miami FL, USA).

\section{Determination of zeta potential}

The stability of DMCNP and CNP was measured by dynamic light scattering using a Zetasizer Nano-ZS (Malvern Instruments, Malvern, UK). Samples of DMCNP and CNP were suspended in filtered distilled water, sonicated, and placed in a disposable cuvette for zeta potential measurements. Nanoparticles were measured for several cycles and comparisons were made based upon their potential and conductivity. Each sample was measured three times and is reported as the mean of triplicate samples.

\section{Morphology and size characterizations}

Morphological analysis and approximation of nanoparticle sizes were confirmed using transmission electron microscopy (TEM) and scanning electron microscopy (SEM), respectively. TEM samples were prepared and visualized as reported. ${ }^{21}$ Similar preparations were made for SEM visualization with a single drop being placed on a glass slide mounted on a SEM stub.

\section{Fourier transform infrared spectra and ultra violet visualization analyses}

Fourier transform infrared spectra (FTIR) were recorded for DMCNP and CNP nanoparticles in attenuated total reflectance (ATR) mode using an IR spectrophotometer (Thermo Fisher Nicolet 380 FT-IR, Thermo Fisher Scientific, Waltham, MA, USA). ${ }^{23}$ The spectra were obtained with 64 scans/sample ranging from 4000 to $400 \mathrm{~cm}^{-1}$ and a resolution of $4 \mathrm{~cm}^{-1}$ and the sample chamber was purged with dry $\mathrm{N}_{2}$ gas. UV-vis was conducted to ascertain the encapsulation of DMOMP in chitosan. Nanoparticles were diluted in deionized water and the absorbance and spectral wavelength were used to determine whether absorption occurred on the outside of the nanoparticle.

\section{Cytotoxicity studies}

Cos-7 cells were purchased from ATCC (Manassas, VA, USA), propagated, and maintained as described. ${ }^{21}$ Cytotoxicity of DMOMP and DMCNP to Cos-7 cells was measured using the 3-(4,5-dimethylthiazol-2-yl)-2,5-diphenyl tetrazolium bromide (MTT) dye reduction assay ${ }^{32}$ and the Cell-Titer 96 Cell Proliferation Assay kit (Promega, Madison, WI, USA). Cells were seeded in a 96-well plate at a density of $10^{5}$ cells/well in $50 \mu \mathrm{L}$ Minimal Essential Media (MEM) (Life Technologies, Carlsbad, CA, USA) supplemented with 10\% Fetal Bovine Serum (FBS) (Life Technologies) and incubated overnight at $37^{\circ} \mathrm{C}$ under $5 \% \mathrm{CO}_{2}$. DMOMP or DMCNP were added in concentrations ranging from 25 to $400 \mu \mathrm{g} / \mathrm{mL}$ and after either 24,48 , or 72 hours, supernatants were removed, cells washed twice with sterile PBS, followed by addition of $15 \mu \mathrm{L}$ of MTT dye solution to each well and cells further incubated for 3 hours at $37^{\circ} \mathrm{C}$ under $5 \% \mathrm{CO}_{2}$. To stop the reaction, $100 \mu \mathrm{L}$ of solubilization solution/stop mixture was added to each well and plates incubated for 30 minutes at room temperature. Absorbance at $570 \mathrm{~nm}$ was measured using a TECAN Sunrise plate reader (TECAN US Inc, Durham, NC, USA). The percentage of cell viability was obtained using the optical density readings of nanoparticle-treated cells compared to those of normal cells (control), where percent viability $=[\mathrm{A}]_{\text {test }} /[\mathrm{A}]_{\text {control }} \times 100$, where $[\mathrm{A}]_{\text {test }}$ is the absorbance of the test sample and $[\mathrm{A}]_{\text {control }}$ is the absorbance of the control sample.

\section{Transfection and Western blot analyses}

Cos-7 cells $\left(1 \times 10^{6}\right.$ cells/well $)$ were transfected by electroporation with DMOMP and DMCNP at concentrations of 2, 5, and $10 \mu \mathrm{g}$ using the Amaxa Cell Line Nucleofector kit-R. Transfected cells were incubated for 48 hours at $37^{\circ} \mathrm{C}$ with $5 \% \mathrm{CO}_{2}$, after which they were washed in PBS and 
fixed using 10\% trichloroacetic acid, followed by successive washing with $70 \%, 90 \%$, and $100 \%$ ethanol with a final wash in $1 \times$ tris buffered saline and tween 20 . Cells were blocked for 30 minutes in blocking buffer $(3 \%$ dry milk in $1 \times$ tris buffered saline), washed in PBS and then incubated for 1 hour with goat anti-C. trachomatis polyclonal antibodies followed by a secondary FITC rabbit anti-Goat $\operatorname{IgG}(\mathrm{H}+\mathrm{L})$ antibody. For some experiments cells were stained with DAPI combined with anti-fade (Invitrogen) mounting solution. Immunofluorescence of cells was visualized using a Nikon Eclipse Ti-U microscope (Nikon Instruments, Melville, New York, USA).

In addition to transfection of Cos-7 cells via electroporation, cells were also chemically transfected using Lipofectamine ${ }^{\mathrm{TM}}$ reagent 2000 (Invitrogen). Cos- 7 cells were seeded $\left(4 \times 10^{5}\right.$ cells/well) in a 6 -well plate for 24 hours after which they were transfected with DMCNP or phCMV 1 vector in opti-MEM transfection media and incubated at $37^{\circ} \mathrm{C}$ for 48 hours. Cell lysates were collected using M-Per mammalian protein extraction reagent (Thermo Fisher Scientific); the resulting lysates were run on an SDS-PAGE gel (Bio-Rad Laboratories, Hercules, CA, USA), transferred onto a PVDF membrane and probed using anti-MOMP polyclonal antibodies (Fitzgerald Industries), followed by an Alexa fluor 680 secondary antibody (Life Technologies). The fixed antibody was viewed using the LI-COR Odyssey imaging apparatus.

\section{Animals}

Six- to eight-week old BALB/c female mice (Charles Rivers, Raleigh, NC, USA) were used for this study. Animal studies were performed following a protocol approved by the Alabama State University (Montgomery, AL, USA) Institutional Animal Care and Use Committee. Mice were housed under standard pathogen-free environmental conditions at ambient temperatures of $25^{\circ} \mathrm{C}$, and provided sterile food and water ad libitum. Mice ( 6 per group) were injected intramuscularly with DMCNP $(80 \mu \mathrm{g} / 200 \mu \mathrm{L}$ of PBS) or with $200 \mu \mathrm{L}$ of PBS. Mice (2 per group) were sacrificed on day 8 and their thigh muscles and spleens were harvested and stored in RNAlater ${ }^{\circledR}$ (Life Technologies) at $-80^{\circ} \mathrm{C}$ until analyzed.

\section{RNA isolation from mouse tissues}

RNA samples were extracted from mouse thigh muscles and spleens using a Qiagen gel extraction kit which included a DNAase-I digestion step according to the manufacturer's protocol. RNA quantifications were made using NanoDrop ND-1000 spectrophotometer at an absorbance of $260 \mathrm{~nm}$. Complementary DNA (cDNA) was produced from quality
RNA using the SuperScript ${ }^{\mathrm{TM}}$ II Reverse Transcriptase kit (Life Technologies) following the manufacturer's protocol. RT-PCR amplification of the MOMP gene transcript was performed using the cDNA as a template, and the same primers as used for cloning.

\section{Results}

\section{Purification and expression of DMOMP}

The major outer membrane protein of C. trachomatis was isolated, amplified through PCR, and cloned into the phCMV1 vector resulting in the vaccine construct, DMOMP. The purified DNA clones as analyzed by restriction enzymes digestion on agarose gel electrophoresis are shown in Figure 1 with the native phCMV1 vector (lane 2), native phCMV1 MOMP (lane 3), phCMV1 vector (4239 bp) restricted BamH 1 and Not 1 (lane 4), phCMV1 MOMP positive clones (4212 bp and $1134 \mathrm{bp}$ ) restricted $\mathrm{BamH} 1$ and Not 1 (lanes 5 and 6) and phCMV1 MOMP clones (lanes 7 and 8) restricted Pst 1 (4823 bp and $531 \mathrm{bp}$ ). Verification of the positive clones was also confirmed by DNA sequencing (Auburn University Genomics and Sequencing laboratory, Auburn, AL, USA).

\section{Structural characterization: FT-IR and UV-vis analyses}

DMOMP was encapsulated in chitosan nanoparticles as illustrated in Figure 2A. A modified method of the complex coacervation technique was used to prepare DMCNP with an achievement of $>90 \%$ encapsulation efficiency. To validate encapsulation of DMOMP in chitosan both FTIR and UV-vis analyses were performed. FTIR analysis of DMOMP indicated peaks at $2039.5 \mathrm{~cm}^{-1}$ and $3737.4 \mathrm{~cm}^{-1}$ representative of the chemical components of the hydroxyl and amine groups (Figure 2B). After encapsulation these chemical signature peaks were no longer detectable, almost providing

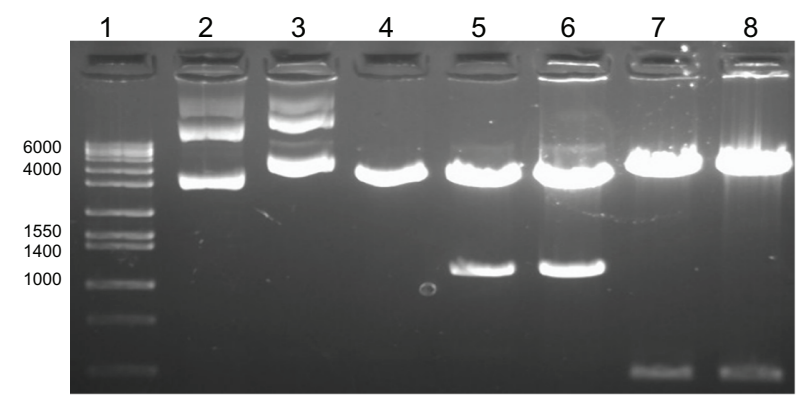

Figure I Agarose gel electrophoresis analysis of DMOMP.

Notes: Molecular weight ( $\mathrm{l} \mathrm{kb}$ ) marker (lane I), native phCMVI vector (lane 2), native phCMVI MOMP (lane 3), phCMVI vector (4239 bp) restricted BamH I and Not I (lane 4), phCMVI MOMP positive clones (42I2 bp and II 34 bp) restricted BamH I and Not I (lanes 5 and 6) and phCMVI MOMP clones (lanes 7 and 8) restricted Pst I (4823 bp and 53I bp). 


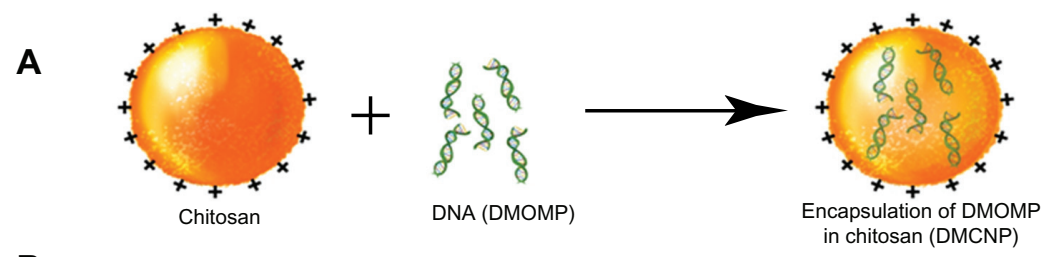

B

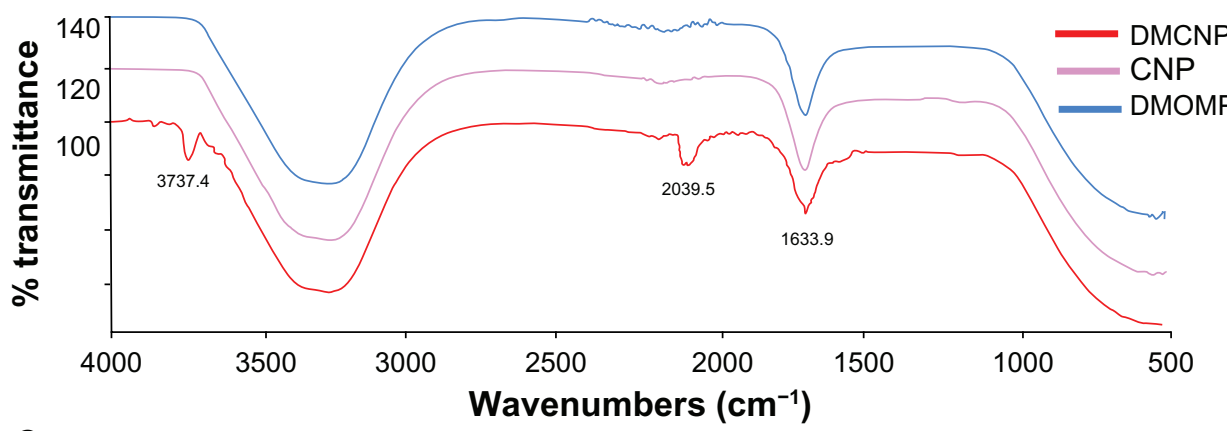

C

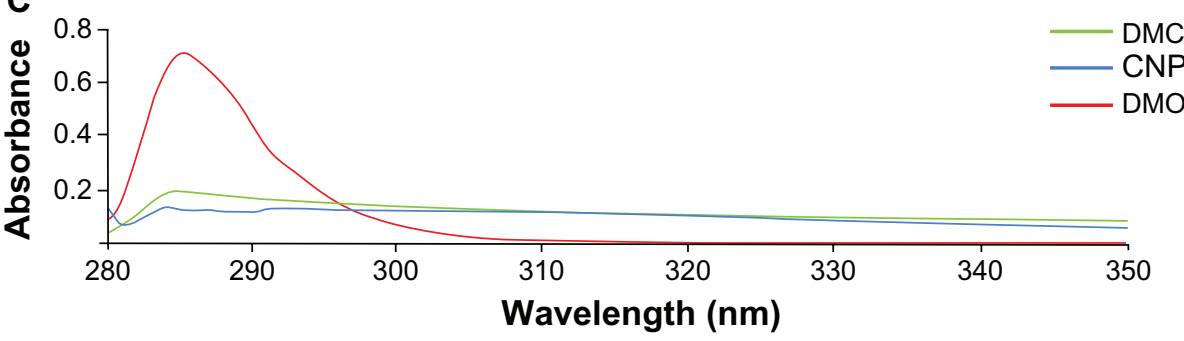

Figure 2 Encapsulation of DMOMP (MOMP DNA) in chitosan nanoparticles. (A) A schematic representation of DMOMP-chitosan nanoparticle construction. (B) Fourier Transfer-Infrared spectroscopy (FT-IR) of DMCNP (DMOMP encapsulated in chitosan nanoparticles), CNP (PBS encapsulated in chitosan nanoparticles) and DMOMP. Recording of the FT-IR spectrum was achieved through 32 scans with a sample ranging from 500 to $4000 \mathrm{~cm}^{-1}$ and a resolution of $4 \mathrm{~cm}^{-1}$ at ambient temperature. Chemical signature peaks observed within the DMCNP spectrum at $2039.5 \mathrm{~cm}^{-1}$ are absent from the DMOMP and CNP spectra. (C) Ultra-violet visible spectra of DMCNP, CNP and DMOMP.

Abbreviations: CNP, phosphate buffered saline encapsulated in chitosan nanoparticles; DMOMP, DNA of the major outer membrane protein of $C$. trachomatis; DMCNP, DMOMP encapsulated in chitosan nanoparticles.

a mirror absorbance image of CNP. These results indicate DMOMP successful encapsulation within the chitosan nanoparticle due to the absence of the aforementioned peaks within the DMCNP profile. UV-vis analysis (Figure 2C) also confirmed the successful encapsulation of DMOMP within the chitosan polymer shell as illustrated by the absorbance readings of DNA at a wavelength of $\sim 285$. In comparison, no absorbance was observed on the surface of DMCNP or CNP further verifying the successful encapsulation of DMOMP within the chitosan nanoparticle.

\section{Size and surface morphology (SEM and TEM) of nanoparticles}

Particle size is a major determinant in the level of mucosal and epithelial tissue uptake of nanostructures, as well as the intracellular interchange of particles. ${ }^{33}$ SEM analysis revealed CNP and DMCNP to be spherical structures (Figure $3 \mathrm{~A}$ and $\mathrm{B}$ ) with sizes of $\sim 50-75 \mathrm{~nm}$ for CNP in comparison to $\sim 167-250 \mathrm{~nm}$ for DMCNP, thus indicating an increase in size due to the encapsulation process. TEM was also employed in performing morphological analysis and size determination of the CNP and DMCNP formulations.
TEM images revealed uniformity in particle size and a homogenous morphology (Figure 3C and D), corroborating the SEM acquired images (Figure 3A and B).
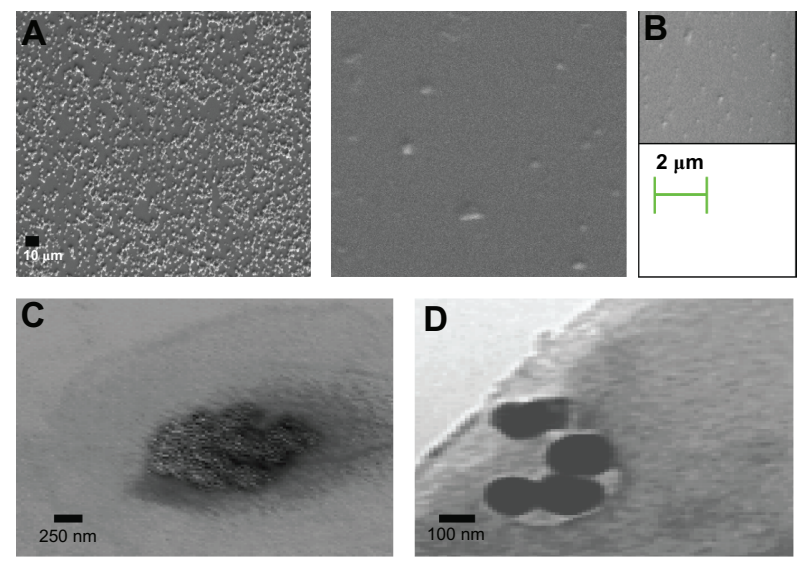

Figure 3 Size and morphological assessments of nanoparticles by SEM and TEM. SEM analyses of CNP (A), DMCNP (B), and TEM analysis of CNP (C) and DMCNP (D). Note: A drop of the nanoparticles was deposited on a copper grid for TEM, or a glass slide mounted on a stub for SEM.

Abbreviations: CNP, phosphate buffered saline encapsulated in chitosan nanoparticles; DMOMP, DNA of the major outer membrane protein of C. trachomatis DMOMP; DMCNP, DMOMP encapsulated in chitosan nanoparticles. 


\section{Cumulative in vitro release of DMOMP from DMCNP}

Release of DMOMP from DMCNP was assessed through incubation of samples in SGF ( $\mathrm{pH}$ 2), SIF ( $\mathrm{pH} 7$ ) and PBS (pH 7.4) over 7 days (Figure 4A). When DMCNP was incubated in SGF, DMOMP was released at a more rapid rate, indicating an increased rate of degradation of chitosan due to its presence in an acidic environment. The release pattern of DMOMP from DMCNP was slow and similar for both SIF and PBS mainly because of the neutral $\mathrm{pH} 7$ solutions, in comparison to that of SGF, whereby DMOMP was released more rapidly at $\mathrm{pH} 2$ (Figure 4A). Products released during the 7-day period were amplified and amplicons verified by agarose gel electrophoresis (Figure 4B). The results show that the released products (Figure 4, lanes 2-6) were indeed DMOMP as validated by the expected size (1154 bp) of the DMOMP positive clone (Figure 4, lane 6).

\section{Stability studies of DMOMP and DMCNP}

Electrophoresis analysis was conducted to assess protection of DMOMP by chitosan nanoparticles. Samples were exposed to chitosanase, DNAase, and restriction enzymes prior to analysis. Electrophoresis analysis of the DMCNP which was not subjected to enzymatic treatments showed no indication of DMOMP released (Figure 5A, lane 2). Following digestion of
DMCNP with chitosanase, the released DMOMP was present in the gel indicating degradation of the nanoparticle by the enzyme (Figure 5A, lane 3). When DMCNP was exposed to both chitosanase and DNAase digestion, no DMOMP was seen in the gel, indicating complete degradation of DNA and chitosan (Figure 5A, lane 4). Simultaneous exposure of DMCNP to chitosanase, restriction enzymes, and DNAase revealed the absence of DMOMP (Figure 5A, lane 5). However, when DMCNP was digested with only restriction enzymes, DMOMP was also not present in the gel, verifying that chitosan surrounds DMOMP, thus preventing restriction enzyme access (Figure 5A, lane 6). DMOMP alone was used as a positive control as validation of the respective product sizes (Figure 5A, lane 8). The findings from this study suggest the capacity of chitosan nanoparticles to protect the encapsulated DMOMP from enzyme degradation.

Stability, integrity, and binding aptitude of the DMCNP in different solutions were examined through an electrophoretic mobility analysis using $1.0 \%$ agarose gel. DMCNP complex disintegration in various $\mathrm{pH}$ solutions is shown in Figure 5B. Stability of the DMCNP was sustained up to $\mathrm{pH} 10$ with no DMOMP being released (Figure 5B). Minor disintegration of DMCNP complexes was observed at $\mathrm{pH} 10.5$ (Figure 5B, lane 9) with a prominent band being observed at $\mathrm{pH} 12$ (Figure 5B, lane 10). These studies show the ability of
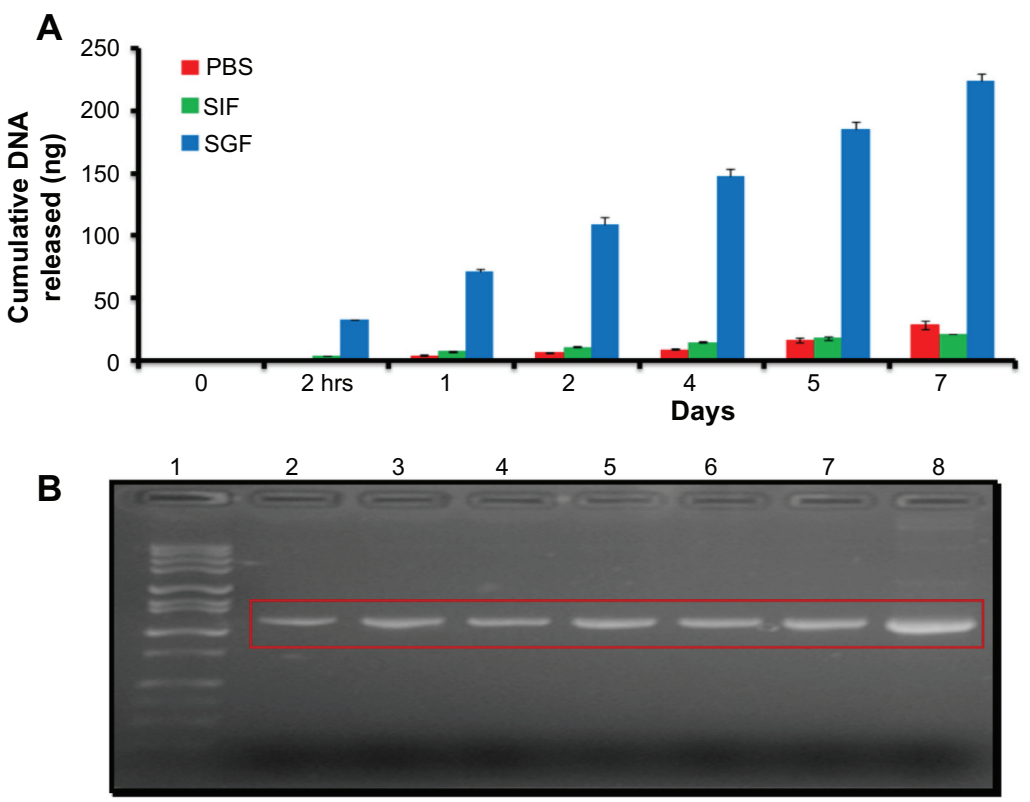

Figure 4 Cumulative DNA release and electrophoretic analysis of released DMOMP. (A) Analysis of encapsulated DMOMP released from DMCNP was evaluated using similar intestinal fluid (SIF, pH 7.0) and similar gastrointestinal fluid (SGF, pH 2.0). Samples were collected at each designated time-interval (2 hours, day I, 2, 4, 5 and 7) and the released DNA measured using NanoDrop at $260 \mathrm{~nm}$. Each bar represents the mean \pm standard deviation of triplicate samples. (B) The released products from each timepoint were precipitated with $5 \mathrm{M} \mathrm{NaCl}$ and amplified by PCR and amplicons subjected to agarose gel electrophoresis for verification of the specific DMOMP product.

Notes: Lanes indicate the sequential collection of amplicons as follows: lane I (I kb marker), lane 2 (2 hours), lane 3 (day I), lane 4 (day 2), lane 5 (day 4), lane 6 (day 5), lane 7 (day 7) and lane 8 (positive DMOMP clone) with an expected size of II 54 bp.

Abbreviations: DMOMP, DNA of the major outer membrane protein of $C$. trachomatis; DMCNP, DMOMP encapsulated in chitosan nanoparticles; hrs, hours; PBS, phosphate buffered saline; PCR, polymerase chain reaction; SGF, similar gastrointestinal fluid; SIF, similar intestinal fluid. 

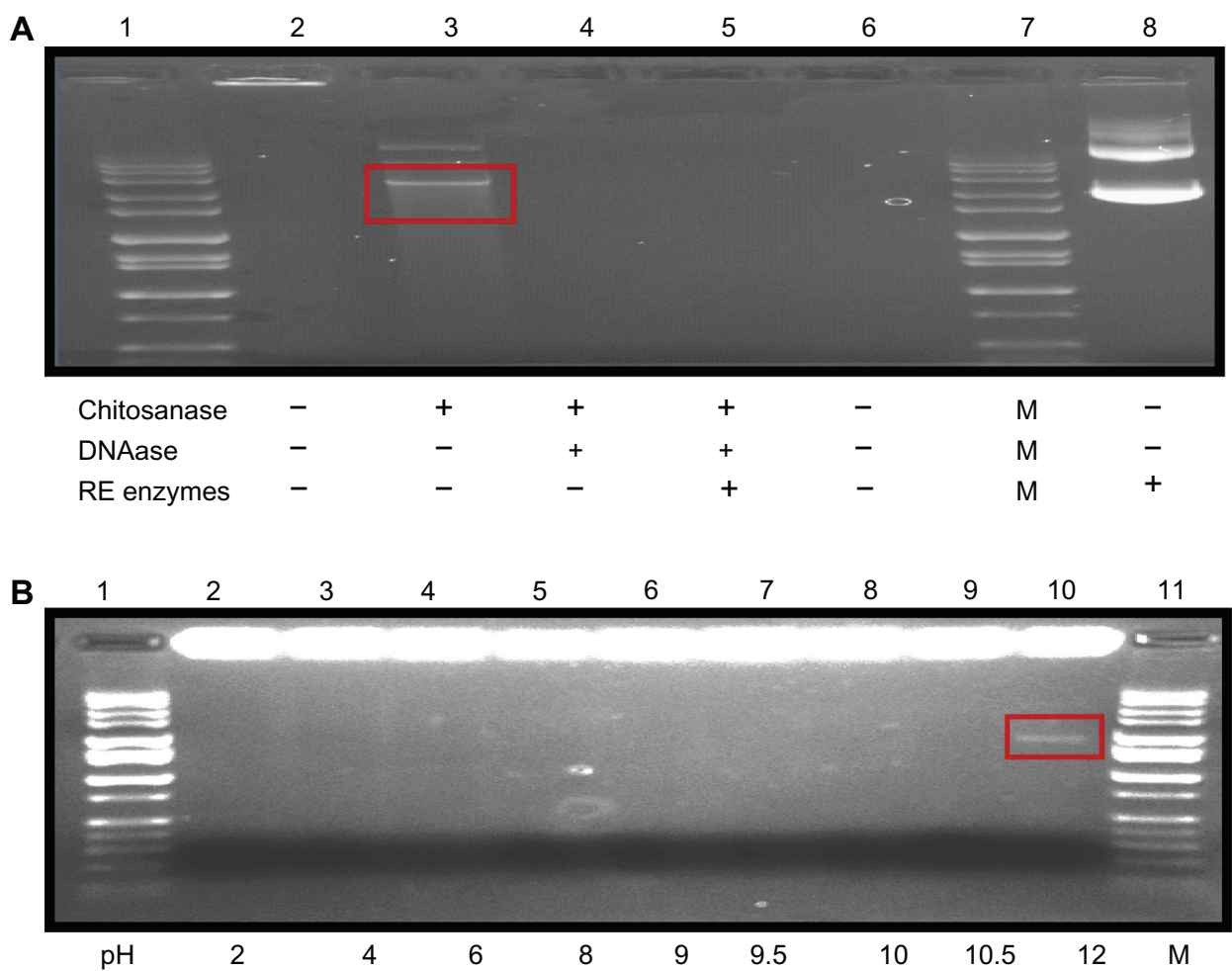

Figure 5 Stability studies of DMOMP in chitosan nanoparticles. (A) Electrophoretic analysis of CNP protection of encapsulated DMOMP after incubation with chitosanase, DNAase I, and restriction enzymes. Both DMCNP and DMOMP $(\mathrm{I} \mathrm{mg} / \mathrm{mL})$ were subjected to enzymatic digestion with Ndel and Sall in combination with, or without chitosanase or with DNAase I. Lane I, (I kb molecular marker), lane 2 (trapped nanoparticle in well), lane 3 (released DMOMP in red box), lanes 4 and 5 (degraded released DMOMP), lane 6 (blank), lane 7 (I kb marker), and lane 8 (DMOMP positive clone). (B) pH stability of encapsulated DMOMP.

Notes: DMCNP was added to individual micro-centrifuge tubes and adjusted to various $\mathrm{pH}$ values $(2,4,6,8,9,10,10.5$ and $\mathrm{I} 2)$ followed by incubation at $37^{\circ} \mathrm{C}$ on a shaker for 30 minutes. Lanes I and I0 are I kb marker. All samples were analyzed by agarose gel electrophoresis and visualized using the Chemilmager gel documentation system. Abbreviations: CNP, phosphate buffered saline encapsulated in chitosan nanoparticles; DMOMP, DNA of the major outer membrane protein of C. trachomatis; DMCNP, DMOMP encapsulated in chitosan nanoparticles; RE, restriction.

chitosan to protect DMOMP from degradation up to $\mathrm{pH} 10$ prior to nanoparticle

\section{Zeta potential}

Assessment of zeta potential is critical in nanoparticle studies, as it may affect both particle stability and mucoadhesion. ${ }^{34}$ The zeta potential value for DMCNP was $8.80 \mathrm{mV}$ in comparison to $0.148 \mathrm{mV}$ for CNP alone (Table 1). These results indicate an increase in stability of DMCNP when compared to that of CNP.

\section{Cytotoxicity studies}

The cytotoxicity of DMCNP and DMOMP was assessed in Cos-7 cells by the MTT assay. Minimal cell death $(>90 \%$

Table I Zeta potential and encapsulation efficiency of nanoparticles

\begin{tabular}{llll}
\hline Nanoparticles & $\begin{array}{l}\text { Size } \\
(\mathbf{n m})\end{array}$ & $\begin{array}{l}\text { Zeta potential } \\
(\mathbf{m V})\end{array}$ & $\begin{array}{l}\text { Encapsulation } \\
\text { efficiency }\end{array}$ \\
\hline CNP & $50-75$ & 0.148 & \\
DMCNP & $167-250$ & 8.80 & $>90 \%$ \\
\hline
\end{tabular}

Abbreviations: CNP, phosphate buffered saline encapsulated in chitosan nanoparticles; DMCNP, DNA of the major outer membrane protein of $C$. trachomatis (DMOMP) encapsulated in chitosan nanoparticles. viable cells) was observed at all concentrations tested from 25 to $400 \mu \mathrm{g} / \mathrm{mL}$ for both DMCNP (Figure 6A) and DMOMP (Figure 6B) and at all examined time-points. Overall no differences were seen on the viability of Cos-7 cells after their exposure to DMCNP and DMOMP.

\section{Transfection studies: gene delivery and protein expression studies}

Qualitative expression of protein upon transfection of Cos-7 cells with DMOMP and DMCNP was evaluated by immunofluorescence microscopy (Figure 7). A level of increase in MOMP protein expression was detected in cells transfected with DMCNP (Figure 7, row B) in comparison to DMOMP (Figure 7, row A). Protein expression was greater for the 5 and $10 \mu \mathrm{g}$ concentrations, than the $2 \mu \mathrm{g}$ concentration, suggesting the ability of chitosan to enhance the level of MOMP protein expression in Cos-7 cells.

Expression in Cos-7 cells was also evaluated through immunofluorescence microscopy using DAPI nuclei stain (Figure 8A) and bright-field (Figure 8B) microscopy. DMCNP-transfected Cos-7 cells were probed with anti-MOMP polyclonal antibodies and as shown in 


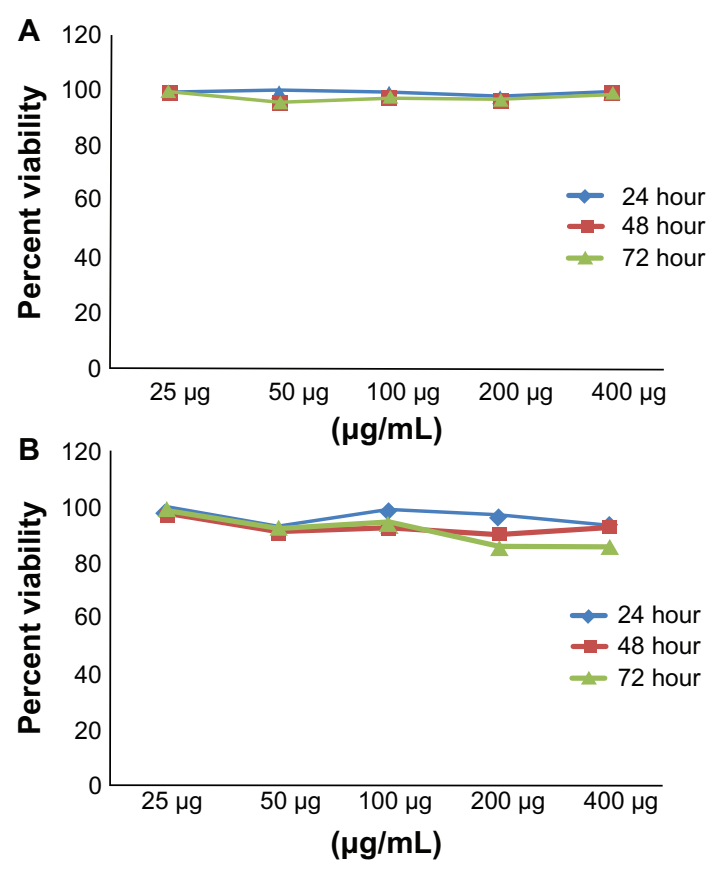

Figure 6 Cytotoxicity analyses of (A) CNP and (B) DMCNP to Cos-7 cells. Notes: Cos-7 cells were seeded in a 96-well plate at a density of $10^{5}$ cells/well/ $50 \mu \mathrm{L}$ in the presence or absence of CNP or DMCNP in concentrations ranging from 25 to $400 \mu \mathrm{g} / \mathrm{mL}$ and incubated at $37^{\circ} \mathrm{C}$ for 24,48 , or 72 hours. The Cell-Titer 96 cell Proliferation Assay kit was used to determine cell viability. Absorbance was read at $570 \mathrm{~nm}$ and \% cell viability was calculated by using the optical density readings compared to normal cells as indicated in the Materials and method section.

Abbreviations: CNP, phosphate buffered saline encapsulated in chitosan nanoparticles; DMCNP, DNA of the major outer membrane protein of $C$. trachomatis (DMOMP) encapsulated in chitosan nanoparticles.

Figure $8 \mathrm{~A}$ and $\mathrm{B}$, MOMP protein expression was clearly observed in the Cos-7 cells exposed to DMCNP as depicted by the green fluorescence cells but not in those exposed to CNP (data not shown). As confirmation of

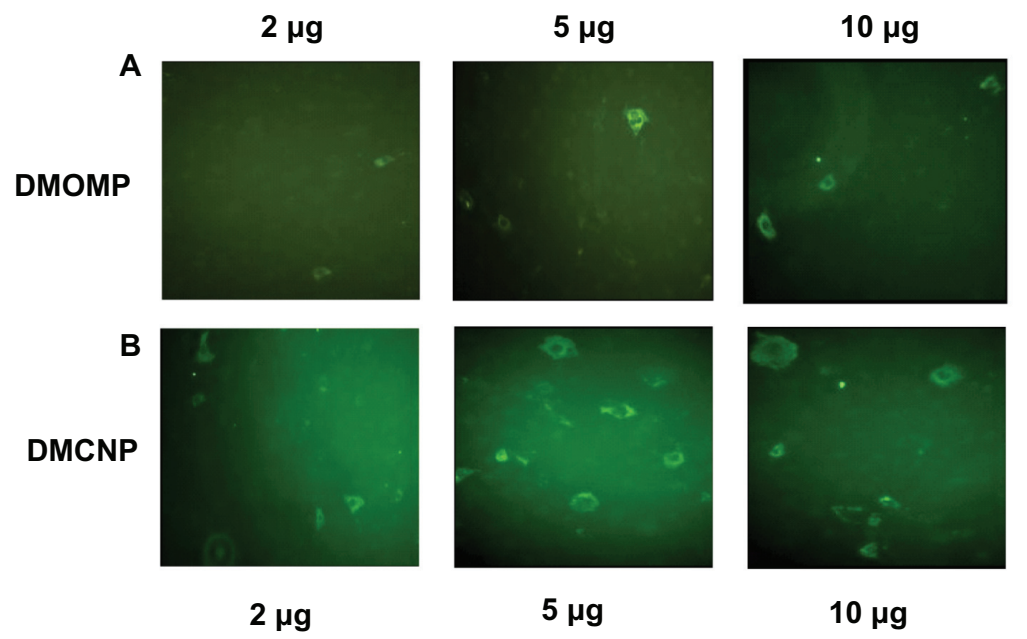

Figure 7 In vitro expression of MOMP protein in transfected Cos-7 cells. Cos-7 cells $\left(1 \times 10^{6}\right.$ cells/well $)$ were transfected by electroporation with DMOMP $(\mathbf{A})$ and DMCNP (B) at concentrations of 2, 5 and $10 \mu \mathrm{g}$.

Notes: Transfected cells were incubated for $48 \mathrm{hrs}$ at $37^{\circ} \mathrm{C}$, fixed and blocked prior to incubation with goat anti-C. trachomatis polyclonal antibodies followed by a secondary FITC rabbit anti-Goat $\operatorname{lgG}(\mathrm{H}+\mathrm{L})$ antibody. Immunofluorescence of cells were visualized using a Nikon Eclipse Ti-U microscope.

Abbreviations: DMOMP, DNA of the major outer membrane protein of $C$. trachomatis; DMCNP, DMOMP encapsulated in chitosan nanoparticles; MOMP, major outer membrane protein of $C$. trachomatis. 


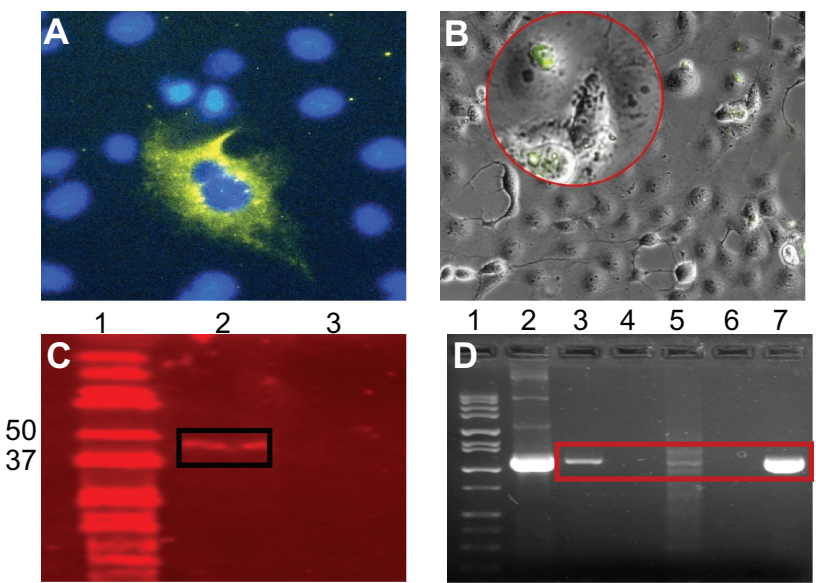

Figure 8 Expression of MOMP at the protein and gene transcript levels. (A) Cos7 cells were transfected as described in Figure 7, immunostained (positive MOMP fluorescence cells) and then mounted with DAPI (blue nuclei stain) combined with an anti-fade mounting solution. (B) Bright-field visualization of Cos-7 cell monolayer showing the MOMP expressed protein. Red circle (positive MOMP fluorescence cells) shows expression of the MOMP protein. (C) Confirmation of expressed MOMP protein by western blot. Cos-7 cells $\left(4 \times 10^{5}\right.$ cells/well) were transfected with DMCNP or phCMVI vector using Lipofectamine and incubated at $37^{\circ} \mathrm{C}$ for 48 hours. Cell lysates were collected, run on an SDS-PAGE gel, transferred onto a PVDF membrane and probed using anti-MOMP polyclonal antibodies followed by an Alexa fluor 680 secondary antibody. The bound antibody was viewed using LI-COR Odyssey imaging apparatus. (D) In vitro expression of MOMP gene transcript. Notes: RNA samples were extracted from mouse thigh muscles and spleens reversed transcribed to cDNA and then subjected to RT-PCR amplification of the MOMP gene transcript using MOMP specific primers. Lane I (MW marker), lanes 2 and 7 (DMOMP positive clones), lane 3 (thigh muscle of DMCNP mice), lane 4 (thigh muscle of PBS mice), lane 5 (spleen from DMCNP mice), and lane 6 (spleen from PBS mice). Red rectangle shows positive MOMP gene transcripts.

Abbreviations: DMCNP, DMOMP encapsulated in chitosan nanoparticles; DMOMP, DNA of the major outer membrane protein of $C$. trachomatis; MOMP, major outer membrane protein of $C$. trachomatis; PVDF, polyvinylidene difluoride.

delivery of the vaccine candidate. Previously we demonstrated the ability of MOMP to induce a balanced $\mathrm{Th}_{1} / \mathrm{Th}_{2}$, serological immune response, ${ }^{9}$ and the capacity of chitosan to enhance delivery and expression of a nano-encapsulated RSV DNA vaccine. ${ }^{21}$ In the present study, we developed a DNA vaccine candidate by cloning the immune-dominant MOMP gene of C. trachomatis into the phCMV1 vector and encapsulating it in chitosan nanoparticles. Our results demonstrate the successful encapsulation and characterization of DMCNP as well as the in vitro and in vivo expression of the MOMP protein and gene transcripts.

In our present study, DMCNPs were produced through the complex coacervation technique due to the mild conditions of the technique, and its successive high encapsulation efficiency rate. Encapsulation of plasmid in chitosan in the generation of a nanoparticle is reliant on various parameters including the N/P ratio (the ratios of moles of the amine groups of cationic polymers to those of the phosphate ones of DNA), polymer charge density, polymer structure, molecular weight of chitosan, degree of deacetylation, and $\mathrm{pH}$ value. ${ }^{35,36}$ This technique allowed us to obtain an encapsulation efficiency of
$90 \%$ or greater which may be due to the addition of sodium acetate to ensure the maintenance of a relatively constant $\mathrm{pH}$, a key parameter in the formation of the encapsulated DMOMP. High rates of encapsulation are achieved when chitosan possessing high levels of deacetylation and increased density of amino acids interacts with phosphate groups present in DNA. ${ }^{37}$ Similar efficiencies have been reported by other investigators who used the complex coacervation technique for encapsulation. ${ }^{21,32,36,38}$

The release profiles of encapsulated DMOMP from chitosan nanoparticles were $\mathrm{pH}$ dependent as revealed by the results obtained in SGF ( $\mathrm{pH}$ 2), SIF ( $\mathrm{pH} 7$ ) and PBS (pH 7). Release profile of any biomaterial from a nanoparticle is an important attribute in nanovaccine development as it can influence the immunological response and immunization protocol. ${ }^{23}$ Within acidic solutions, the amino acid groups of chitosan are protonated with the resultant soluble polysaccharide being charged. ${ }^{39}$ Studies by $\mathrm{Hu}$ et $\mathrm{al}^{40}$ demonstrated that the release of a gene from the nanoparticle depends greatly on the swelling of the nanoparticle and $\mathrm{pH}$ values of the releasing solution. Consistent with previous studies our results indicate that the release pattern of encapsulated DMOMP was biphasic $^{21}$ with an initial burst preceding a controlled release which was also $\mathrm{pH}$ dependent. ${ }^{40}$

The initial release of DMOMP observed in all three $\mathrm{pH}$ solutions could be attributed to the release of molecules weakly associated with the chitosan nanoparticles located at, or near the nanoparticle surface, whereas the second phase of the release profile may correspond to DNA molecules more proficiently entrapped within the nanoparticle. In our study, different release profiles were obtained for DMCNP and were dependent on its presence in either an alkaline or acidic solution. At $\mathrm{pH}$ values of 7 to 7.4 , chitosan nanoparticles are comparatively more swollen than those at a $\mathrm{pH}<4.0,{ }^{40}$ resulting in a slower release of encapsulated DNA due to slower degradation of the nanoparticles. The release of encapsulated DMOMP in SIF and PBS solutions was less due to chitosan inability to solubilize in neutral solutions. In comparison, DMCNP exposed to SGF released its entrapped DMOMP at a more rapid pace due to excessive degradation of the chitosan nanoparticle in a more acidic solution.

Here our analysis demonstrates the ability of chitosan to protect encapsulated DMOMP from degradation, corroborating previous reports. ${ }^{21,35,41}$ Our results clearly indicate that release of DMOMP was enzyme-dependent and that our chitosan nanoparticle exhibits protective properties promoting its potential as an effective and efficient transport system capable of safe delivery and protection of DMOMP 
from degradation by biological enzymes. Within cells, chitosan is degraded by lysozyme and N-acetyl-glucose amidase, both of which are present in the endosomal vesicle. The presence of these two enzymes indicates that degradation and the ensuing release of the plasmid will begin immediately following endocytosis. ${ }^{41}$

In our study DMCNP nanoparticles ranged from $167-250 \mathrm{~nm}$ in diameter and their shapes were spherical with a homologous morphology. It is known that nanoparticles smaller than $10 \mu \mathrm{m}$ are phagocytized by antigen presenting cells at the mucosal surfaces, leading to a potency increase, or potentiating immune responses. ${ }^{42,43}$ According to Boyoglu et al, ${ }^{21}$ the physical state and stability of the nanoparticle can be considered key parameters for efficient and effective peptide delivery. Thus, DMCNP produced through our modified complex coacervation technique possessed the required size necessary to facilitate uptake by APCs, which is necessary for induction of immune responses.

We employed the MTT test to assess the overall effects of DMOMP and DMCNP on the metabolic activity of Cos-7 cells. Our results overall showed no cytotoxic effects of DMOMP or DMCNP for Cos-7 cells as cell viabilities remained greater than $90 \%$. Taking into consideration that proliferating cells are metabolically more active than nonproliferating cells, results obtained can also be interpreted as the possible impact of DMOMP and DMCNP solutions on cellular proliferation. This is perhaps indicative that polymers may favorably manipulate both cellular lysosomal and mitochondrial activity. ${ }^{44}$

Since its initial usage as a vector-based delivery system for the transport of genes into the cellular matrix, ${ }^{45}$ chitosan and its derivatives have revealed immense promise for encapsulation of DNA. In our study we demonstrated that chitosan not only protected, but also enhanced expression of DMOMP. Previous studies have shown that chitosan possesses capabilities of opening tight cellular junctions through varying effects upon F-actin filaments. ${ }^{46}$ This, along with the small size of chitosan nanparticles being engulfed by endocytosis into endocytic vesicles, allows it entry into targeted cells via transferring cytosis, ${ }^{47}$ which may explain the enhanced effect of DMCNP on encapsulated DMOMP delivery into both Cos-7 cell monolayers and animal tissues within our study. Also, as cell culture substrates, chitosan and its derivatives may allow activation of varying endocytic pathways thus increasing nanoparticle internalization or plasmid tranfection. ${ }^{48}$ Saka and Bozkir $^{49}$ reported increased levels of transfection efficiency in vitro using chitosan-based nanoparticle formulations, thereby corroborating our results in the present study.
Here in the present study expression was seen not only in Cos-7 cells but also in mice. Expression of the MOMP gene was observed to be localized as well as systemic, respectively in thigh muscles and spleens of mice. Increased levels of gene expression have been demonstrated through chitosan-plasmid usage in comparison to plasmid usage alone. ${ }^{50}$ Reportedly, Jean and colleagues, ${ }^{50}$ demonstrated encapsulation of a type 2 diabetes gene for treatment of mice produced results two-fold greater than plasmid administration alone. Our results strongly suggest that DMCNP can improve DMOMP expression with enhanced delivery to targeted tissues.

\section{Conclusion}

Our study, to the best of our knowledge, is the first to encapsulate a phCMV1-MOMP DNA construct in chitosan, perform physiochemical characterizations, and assess its expression by both in vitro and in vivo experiments. In our study, DMCNP was generated through the complex coacervation technique with $>90$ encapsulation efficiency. DMCNP was 167-250 nm and spherical in shape. Notably, chitosan protected DMOMP from degradation and enhanced its protein expression in Cos-7 cells. More significantly, the MOMP gene transcript was expressed locally and systemically in tissues of mice. Our data accentuate the efficiency of chitosan as a delivery vehicle for the formulation of a $C$. trachomatis vaccine candidate. In addition, DMCNP has the potential of a promising nano-encapsulated vaccine formulation and warrants further studies in mice.

\section{Acknowledgment}

This research was supported by funding from National Science Foundation (NSF) grants NSF-CREST (HRD-1241701) and NSF-HBCU-UP (HRD-1135863). We thank Drs Mamie Coats and Saurabh Dixit for their help with the mice study, Mr Elijah Nyairo for assistance with the FTIR, and Dr Michael Miller, Auburn University, for help with TEM and SEM images. Special thanks go to Eva A Dennis for Figure 2A illustration, and to the Center for NanoBiotechnology Research (CNBR) staff, Yvonne Williams and Lashaundria Lucas for their time and support.

\section{Disclosure}

The authors report no conflict of interests in this work.

\section{References}

1. World Health Organization. Global Prevalence and Incidence of Selected Sexually Transmitted Diseases: Overviews and Estimates. Geneva, Switzerland: World Health Organization; 1996:1-52. 
2. World Health Organization. DoRHaR, Prevalence and Incidence of Selected Sexually Transmitted Infections. Geneva, Switzerland: World Health Organization; 2011.

3. Westrom LJR, Joesoef R, Reynolds G, Hagdu A, Thompson SE. Pelvic inflammatory disease and fertility. A cohort study of 1,844 women with laparoscopically verified disease and 657 control women with normal laparoscopic results. Sex Transm Dis. 1992;4:185-192.

4. Plummer FA, Simonsen JN, Cameron SJ, Ndinya-Achola JO, Kreiss JK, Gakinya NM. Cofactors in male-female sexual transmission of human immunodeficiency virus type 1. J Infect Dis. 1991;6:1236-1237.

5. Beagley KW, Timms P. Chlamydia trachomatis infection: incidence, health costs and prospects for vaccine development. J Reprod Immunol. 2000;48:47-68.

6. Karunakaran KP, Yu H, Foster LJ, Brunham RC. Development of a Chlamydia trachomatis T cell vaccine. Hum Vaccine. 2010;6:676-680.

7. Caldwell D, Kromhout J, Schachter J. Purification and partial characterization of the major outer membrane protein of Chlamydia trachomatis. Infect Immun. 1981;31:1161-1176.

8. Coler RN, Bhatia A, Maisonneuve JF, et al. Identification and characterization of novel recombinant vaccine antigens for immunization against genital Chlamydia trachomatis. FEMS Immunol Med Microbiol. 2009;55:258-270.

9. Singh SR, Hulett K, Pillai SR, Dennis VA, Oh MK, Scissum-Gunn K. Mucosal immunization with recombinant MOMP genetically linked with modified cholera toxin confers protection against Chlamydia trachomatis infection. Vaccine. 2006;24:1213-1224.

10. Ortiz L, Angevine M, Kim SK, Watkins D, DeMars R. T-cell epitopes in variable segments of Chlamydia trachomatis major outer membrane protein elicit serovar-specific immune responses in infected humans. Infect Immun. 2000;68:1719-1723.

11. Stagg AJ, Elsley WA, Pickett MA, Ward ME, Knight SC. Primary human T-cell responses to the major outer membrane protein of Chlamydia trachomatis. Immunology. 1993;79:1-9.

12. Zhu S, Chen J, Zheng M, Gong W, Xue X, Li W. Identification of immunodominant linear B-cell epitopes within the major outer membrane protein of Chlamydia trachomatis. Acta Biochim Biophys Sin. 2010;42:8.

13. Xu W, Liu J, Gong W, Chen J, Zhu S, Zhang L. Protective immunity against Chlamydia trachomatis genital infection induced by a vaccine based on the major outer membrane multi-epitope human papillomavirus major capsid protein L1. Vaccine. 2011;29:2672-2678.

14. Pal S, Peterson EM, de la Maza LM. Vaccination with the Chlamydiatrachomatis major outer membrane protein can elicit an immune response as protective as that resulting from inoculation with live bacteria. Infect Immun. 2005;73:8153-8160.

15. Sun G, Pal S, Weiland J, Peterson EM, de la Maza LM. Protection against an intranasal challenge by vaccines formulated with native and recombinant preparations of the Chlamydia trachomatis major outer membrane protein. Vaccine. 2009;27:5020-5025.

16. Eko FO, He Q, Brown T, et al. A novel recombinant multi-subunit vaccine against Chlamydia. J Immunol. 2004;173:3375-3382.

17. Dai C, Wang B. Microencapsulation peptide and protein drugs delivery system. Colloid Surf B Biointerfaces. 2005;41:117-120.

18. Tyagi RK, Garg NK, Sahu T. Vaccination strategies against malaria: novel carrier(s) more than a tour de force. J Control Release. 2012;162: 242-254.

19. Kathuria N, Kraynyak KA, Carnathan D, Betts M, Weiner DB, Kutzler MA. Generation of antigen-specific immunity following systemic immunization with DNA vaccine encoding CCL25 Chemokine Immunoadjuvant. Hum Vaccin Immunother. 2012:8;1607-1619.

20. Childs TS, Webley WC. In vitro assessment of halobacterial gas vesicles as a Chlamydia vaccine display and delivery system. Vaccine. 2012;30: 5942-5948.

21. Boyoglu S, Vig K, Pillai S, Rangari V, Dennis VA, Khazi F. Enhanced delivery and expression of a nanoencapsulated DNA vaccine vector for respiratory syncytial virus. Nanomedicine. 2009;5: $463-472$.
22. Tyagi RK, Sharma PK, Vyas SP, Mehta A. Various carrier system(s)mediated genetic vaccination strategies against malaria. Expert Rev Vaccines. 2008;7:499-520. Retraction in Expert Rev Vaccines. 2011;10:1244.

23. Taha MA, Singh SR, Dennis VA. Biodegradable PLGA85/15 nanoparticles as a delivery vehicle for Chlamydia trachomatis recombinant MOMP-187 peptide. Nanotechnology. 2012;23:325101.

24. Felt O, Buri P, Gurny R. Chitosan: a unique polysaccharide for drug delivery. Drug Dev Ind Pharm. 1998;24:979-993.

25. MacLaughlin FC, Mumper RJ, Wang J. Chitosan and depolymerized chitosan oligomers as condensing carriers for in vivo plasmid delivery. J Controlled Rel. 1998;56:259-272.

26. Mao S, Shuai X, Unger F, Simon M. The depolymerization of chitosan: effects on physiochemical and biological properties. Int $J$ Pharm. 2004:281:45-54.

27. Gan Q, Wang T, Cochrane C, McCarron P. Modulation of surface charge, particle size and morphological properties of chitosan-TPP nanoparticles intended for gene delivery. Colloids Surf B Biointerfaces. 2005;44:65-73.

28. Janes KA, Calvo P, Alonso MJ. Polysaccharide colloidal particles as delivery systems for macromolecules. Adv Drug Deliv Rev. 2001;47:83-97.

29. Artursson P, Lindmark T, Davis SS, Illum L. Effect of chitosan on the permeability of monolayers of intestinal epithelial cells (Caco-2). Pharm Res. 1994;11:1358-1361.

30. Gan Q, Wang T. Chitosan nanoparticles as protein delivery carrierSystematic examination of fabrication conditions for efficient loading and release. Colloids Surf B Biointerfaces. 2007;59:24-34.

31. Sun Y, Zhang S, Peng X, et al. Preparation, characterization and transfection efficacy of chitosan nanoparticles containing the intestinal trefoil factor gene. Mol Biol Rep. 2012;39:945-952.

32. Yu-Hong L, Min-Wen F, Zhi C, Qi Z, Hai-Rui Y. Chitosan-DNA microparticles as mucosal delivery system: synthesis, characterization and release in vitro. Chin Med. 2005;118:936-941.

33. Panyam J, Labhasetwar V. Biodegradable nanoparticles for drug and gene delivery to cells and tissues. Adv Drug Deliv Rev. 2003;55: 329-347.

34. Ravi Kumar MN, Bakowsky U, Lehr CM. Preparation and characterization of cationic PLGA nanospheres as DNA carriers. Biomaterials. 2004;25:1771-1777.

35. Bozkir A, Saka OM. Chitosan-DNA nanoparticles: effect on DNA integrity, bacterial transformation and transfection efficiency. $J$ Drug Target. 2004;12:281-288.

36. Mao HQ, Roy K, Troung-Le VL, Janes KA, Lin KY, Wang Y. Chitosan-DNA nanoparticles as gene carriers: synthesis, characterization and transfection efficiency. $J$ Control Release. 2001;70:399-421.

37. Borchard G. Chitosans for gene delivery. Adv Drug Deliv Rev. 2001;52: 145-150.

38. Zhao K, Shi X, Zhao Y, et al. Preparation and immunological effectiveness of a swine influenza DNA vaccine encapsulated in chitosan nanoparticles. Vaccine. 2011;29:8549-8556.

39. Hejazi R, Amiji M. Chitosan-based gastrointestinal delivery systems. J Control Release. 2003;89:151-165.

40. Hu Y, Jiang X, Ding Y, Ge H, Yuan Y, Yang C. Synthesis and characterization of chitosan-poly(acrylic acid) nanoparticles. Biomaterials. 2002;23:3193-3201.

41. Köping-Höggård $\mathrm{M}$, Tubulekas I, Guan H, Edwards K, Nilsson M, Vårum KM. Chitosan as a non-viral gene delivery system. Structureproperty relationships and characteristics compared with polyethylenimine in vitro and after lung administration in vivo. Gene Ther. 2001;8:1108-1121.

42. Waeckerle-Men Y, Groettrup M. PLGA microspheres for improved antigen delivery to dendritic cells as cellular vaccines. Adv Drug Deliv Rev. 2005;57:475-482.

43. Waeckerle-Men Y, Allmen EU, Gander B, et al. Encapsulation of proteins and peptides into biodegradable poly(d,l-lactide-co-glycolide) microspheres prolongs and enhances antigen presentation by human dendritic cells. Vaccine. 2006;24:1847-1857. 
44. Guerra GD, Cerrai P, Tricoli M, Maltini S, del Guerra RS. In-vitro cytotoxicity testing of chitosan-containing polyelectrolyte complexes. J Mater Sci Mater Med. 1998;9:73-76.

45. Mumper RJ, Claspell JM, Rolland AP. Novel polymeric condensing carriers for gene delivery. Proc Natl Acad Sci U S A. 1995;22: $178-179$.

46. Illum L, Jabbal-Gill I, Hinchcliffe M, Fisher AN, Davis SS. Chitosan as a novel nasal delivery system for vaccines. Adv Drug Deliv Rev. 2001;51:81-96.

47. Vinogradov SV, Kabanov AV. Nanosized cationic hyrogels for drug delivery: preparation, properties and interactions with cells. Adv Drug Deliv Rev. 2002;54:135-147.
48. Hsu SH, Ho TT, Tseng TC. Nanoparticle uptake and gene transfer efficiency for MSCs on chitosan and chitosan-hyaluronan substrates. Biomaterials. 2012;33:3639-3650.

49. Saka OM, Bozkir A. Formulation and in vitro characterization of PEGylated chitosan and polyethylene imine polymers with thrombospondin-I gene bearing pDNA. J Biomed Mater Res B Appl Biomater. 2012;100:984-992.

50. Jean M, Alameh M, Buschmann MD, Merzouki A. Effective and safe gene-based delivery of GLP-1 using chitosan/plasmid-DNA therapeutic nanocomplexes in an animal model of type 2 diabetes. Gene Ther 2011;18:807-816.
International Journal of Nanomedicine

\section{Publish your work in this journal}

The International Journal of Nanomedicine is an international, peerreviewed journal focusing on the application of nanotechnology in diagnostics, therapeutics, and drug delivery systems throughout the biomedical field. This journal is indexed on PubMed Central,

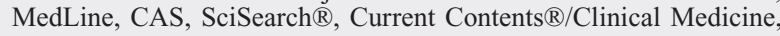

\section{Dovepress}

Journal Citation Reports/Science Edition, EMBase, Scopus and the Elsevier Bibliographic databases. The manuscript management system is completely online and includes a very quick and fair peer-review system, which is all easy to use. Visit http://www.dovepress.com/ testimonials.php to read real quotes from published authors.

Submit your manuscript here: http://www.dovepress.com/international-journal-of-nanomedicine-journal 